\title{
Teaching Industrial Automation Concepts with the use of Virtual/Augmented Reality - The IEC 61499 Case
}

\author{
Christos Chrysoulas \\ School of Engineering, Computer \\ Science and Informatics Dep. London \\ South Bank Univeristy London, UK \\ chrysouc@1sbu.ac.uk
}

\author{
Aydin Homay \\ R\&D PEER Group GmbH \\ Dresden, Germany \\ homay@ieee.org
}

\author{
Maria Lemac \\ School of Engineering, Computer \\ Science and Informatics Dep. London \\ South Bank Univeristy London, UK \\ lemacm@1sbu.ac.uk
}

\begin{abstract}
We propose a framework for teaching complex industrial automation concepts with the help of Virtual/Augmented reality. Initially we construct the needed 3D models like conveyors belts, and liquid tanks to be used as testbed examples for the proposed approach and afterwards with the use of the appropriate engine and the Virtual Reality headset we create the enriched virtual and augmented reality use cases to support higher education students better understand complex industrial automation concepts. As this is an initial framework proposal, an extended literature review providing the needed background information on the status and progress of the Virtual and Augmented reality for educational and not only purposes is provided. The proposed system's architecture is in detail explained and use cases where the proposed approach can be used are presented. An overview of the IEC 61499 framework is also presented since it is the underlying framework for supporting the industrial automation concepts.
\end{abstract}

Keywords-virtual reality, augmented reality, industrial automation, education, IEC 61499

\section{INTRODUCTION}

Virtual reality technology can make a significant impact on the learning process. Although we are seeing some innovative changes in education, it has not changed for years in terms of teaching approaches and techniques used. Research on the Internet and use of instructional videos have become part of regular teaching and distance learning. However, Virtual Reality is yet to become a part of the teaching and learning process. VR supports a constructivist approach to learning as it allows a learner to learn by doing.

Broadly looking at the benefits of using VR in teaching and learning, we could identify two main aspects. One aspect is the capability of the VR technology to create an environment that allows the students to visualise, examine and experience reality, this greatly aids complex conceptual learning. Another aspect is related to the student motivation, it encourages active participation rather than passivity and allows the learner to proceed through an experience at their own pace as the time does not need to be fixed by a regular class schedule.

It is possible to more accurately and, in more detail, illustrate some features, processes and complex constructs with Virtual Reality than by other means. VR allows extreme closeup examination of an object and gives the opportunity for new insights. In addition to this, VR holds the attention of students.
Students find it exciting and challenging to walk through an environment in three dimensions and interact with it.

From a systems engineering industry point of view, automation is a core element in recent years. Obviously, there is a special interest in certain aspects, such as the knowledge of the state of many variables within a set of possible values or the state of a process associated with a finite number of situations. Thus, sensors, actuators and controllers are required to manage all subsystems. Control Engineering studies often require expensive equipment as well as access to the real infrastructure to provide experimental evaluation of theoretical concepts. The investment is usually a limitation for many academic institutions. Such situation has provoked an increasing interest in developing virtual industrial plants to help students interact with a quasi-real automated environment. The development of virtual laboratories has been a core issue in engineering education.

We propose a framework for teaching complex industrial automation concepts with the help of Virtual/Augmented reality. Initially we are constructing some three-dimensional models representing real world objects such as liquid tanks and conveyors belts to be used as testbed examples for the proposed approach.

The IEC 61499 [1] framework comes with its own tools for building automations systems. Namely FORTE [2] and 4DIAC [2]. FORTE is basically a virtual machine that executes IEC 61499 applications. This execution environment is developed together with the 4DIAC development environment, where the programmer may design IEC 61499 applications using a graphical interface. Once programmed, 4DIAC downloads the IEC 61499 applications onto IEC 61499 execution environments using a standardized interface of these execution environments.

Notice that when an application is downloaded from 4DIAC onto the execution environment, what is in fact downloaded is a list of FBs that need to be instantiated, and how the data and event interfaces of these FBs are to be interconnected. Since the actual implementation of basic FBs is not downloaded, this means that a program developer that decides that (s)he needs to implement a new basic FB, that basic FB must be compiled and linked into the execution environment before the IEC 61499 application using that basic FB can be downloaded to that same execution environment. 
Applications in IEC 61499 can be easily described as being composed by a network of blocks that transfer data and events between them. This building block, known as a Function Block (FB), has data and event inputs as well as data and event outputs. This FB network is typically drawn in a graphical editor, and an IEC 61499 application will include the data and event connections between the FBs.

The idea behind the proposed framework is to provide the ability to a Virtual/Augmented Reality user/student to easily build the automation system though the already provided IEC 61499 tool (FORTE and 4DIAC) and also provide him/her with the ability to parameterise the FBs' parameters to serve his/her needs. Another aspect is the capability to visualize the results with the use of examples available in a well build library. Examples like conveyors belt control [3] and level control in a liquid's tanks.

In order the above to be feasible, the needed interfaces for the communication among the Virtual/Augmented Reality software and the IEC 61499 framework should be designed and developed and also the interfaces for the communication of the IEC 61499 to the actual use-cases should take place.

The current work will be a first attempt to present a thorough state of the art review on what is available today regarding the use of Virtual/Augmented Reality in the educational discipline and present an initial framework that can be applied for teaching (complex) industrial automation concepts in higher education.

The rest of the paper is organised as follows: Section II gives an insight on existing techniques on virtual and augmented reality in education. Section III presents the proposed system's architecture, and uses case for the validation are presented. Section IV concludes this work and provides future plans.

\section{RELATED WORK}

The Medical Virtual Reality group at the University of Southern California Institute for Creative Technologies studies use cases of virtual reality simulation technology for clinical purposes [4]. VR environment is also used for training purposes in the military, which includes flight and battlefield simulations, medical training under battlefield conditions, virtual boot camp and similar.

In [17], the authors provide a more a practical approach for engaging students in the learning process. During a 6-week introductory class project, first year students of the Master in Civil Engineering will create virtual environments in which the models are created in CAD drawings and imported into Unity3D. Augmented reality is also implemented for mobile device to recognize the image of an object. This is very interesting approach since it helps students engage in the whole process, from creating a model, incorporating the model into Unity3D and exporting results to VR/AR headset devices. This report does not provide much insight of how to build the application from a technical perspective, so it is a bit difficult to reconstruct the learning process.
Kaufmann [5] analysed the effectiveness of VR tools in teaching spatial perception, spatial visualization, mental rotations, spatial relations and spatial orientation. He identified that the main advantage of VR to student learning in mathematics and geometry education is that students actually see three dimensional objects which they otherwise need to calculate and construct with traditional methods. He stated that "Virtual reality provides them with a nearly tangible picture of complex three-dimensional objects and scenes. It enhances, enriches and complements the mental pictures that students form in their minds when working with three dimensional objects." He concluded that by working directly in 3D space, complex spatial problems and relationships can be comprehended better and faster than with traditional methods.

Chen et al. [12] in their work provide an AR-based system to increase the spatial awareness and interest of learning for Engineering Graphics Education. 3D virtual objects are overlaid on 2D drawing to help students quickly access to 3D solid structure and spatial detail information. The proposed approach significantly reduces the time spent by the instructors and students in the classroom. Messner et al. [13] provide an expensive approach by using the CAVE-like projection system for teaching undergraduate Architectural Engineering students how to create VR interfaces. The proposed approach proven to have a really big influence on the student performance since students were able to develop a plan of the construction of a nuclear power plant within a short time (around one hour) even though their limited experience in buildings and infrastructures.

Mantovani [6] identified that visualization and reification, an alternate method for presentation of material; learning in contexts impossible or difficult to experience in real life; motivation enhancement; collaboration fostering; adaptability, offering the possibility for learning to be tailored to learner's characteristics and needs were the potential benefits of the use of VR in education and training.

Serafin et al. [14] provided a number of approaches on how to properly integrate virtual and augmented reality in the musical education from both an academic and a commercial perspective. The study showed that the quality of learning and the retention rate of students in the classroom had improved significantly. Many potential use cases where the proposed approach could be applied also proposed. The paper only points out some available approaches and promising research directions without any further guiding on how to build a real application.

Parmar et al. [15], based their work on the similarities of dance with programming, thus creating a Virtual Environment Interactions application in order students to learn complicated concepts of programming (sequences, loops, conditionals, variables, functions, etc). The results derived from this study showed that students are better engaging by learning activities and remembered what they saw in the VR context rather than the knowledge received by purely laboratory based tutoring.

Salzman et al, [7] describe how virtual reality aids complex conceptual learning, and how virtual reality's features and other factors shape the learning process and learning outcomes. Chee [8] argues for the need to root learning in experience, using physics as an example. He states that physics students have 
little "feel" and "understanding of the qualitative dimensions of the phenomena they study". Chee believes that virtual reality can be used to "provide a foundation for students' conceptual and higher-order learning".

Zacharia [9] confirms that "researchers attribute success of simulations to the empowerment of students, the unique instructional capabilities, the support for new instructional approaches, the development of cognitive skills, and the development of attitudes".

Pantelidis [10] states that VR can change the way a learner interacts with the subject matter as it requires the students to actively participate and they can see the results immediately. VR provides an opportunity for the learner to make discoveries previously unknown. New perspectives are made possible by modelling the real world, and studying the model can provide insights never before realized. VR also allows the disabled to participate in an experiment or learning environment when they cannot do so otherwise.

\section{SYSTEM ARCHITECTURE}

In this section we propose a framework based on IEC 61499 and Unity 3D engine for building industrial automation applications enriched with the virtual/augmented reality concept. The proposed architecture, as depicted in Fig.1 includes four main components. Namely: (i) FORTE; (ii) 4DIAC; (iii) Virtual Reality Environment (Unity 3D); and (iv) Use cases (Conveyors belts, liquid tanks, etc.). the following subsections provide an in depth information for each component.

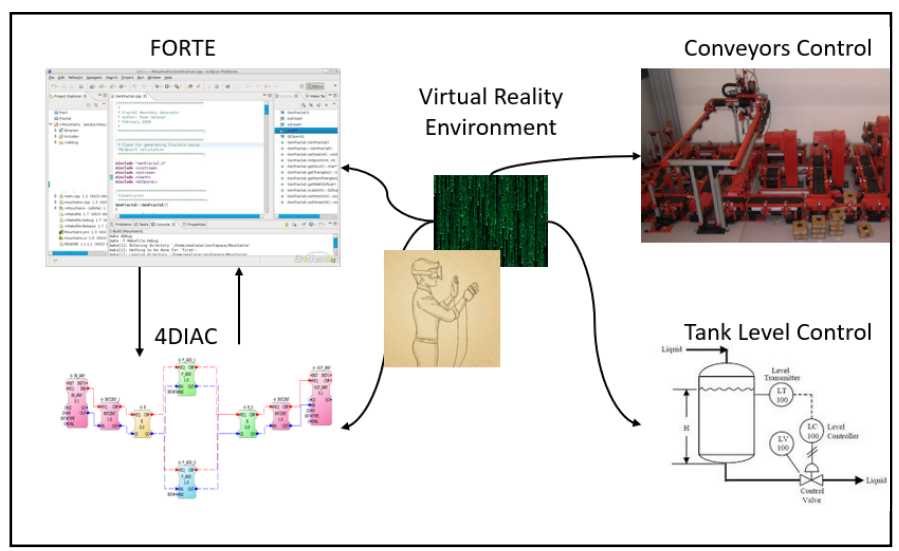

Fig. 1. Proposed Abstarct Architecture

\section{A. IEC 61499 Framework}

IEC 61131 [18] has been widely accepted in the industrial automation domain. Despite its great acceptance, it is claimed that the standard does not address the new requirements and characteristics of today's complex industrial systems. Characteristics like: (i) portability, (ii) interoperability, (iii) increased reusability and (iv) distribution. To cope these restrictions, IEC has initiated the task of developing IEC 61499 , which is nowadays presented as a mature technology to enable intelligent automation in various domains. IEC identified the need for developing a new function block standard with the idea of using these function blocks in a wide range for distributed industrial process control system. They tried to develop something enough generic to be usable in the majority of industries. Therefore, the IEC 61499 is more capable and flexible than IEC 61131 (the first standard for industrial automation system) [11].

The IEC 61499 standard should not consider a programming methodology, but rather a framework to describe the architecture. The IEC 61499 standard is a modeling language based on event-driven function blocks, and models targeting the component-oriented distributed systems world. The IEC 61499 standard developed to meet the requirements of intelligent automation using model-driven engineering; it uses function blocks as its basic design entities. The IEC 61499 applications are composed of Function Block (FB) networks that encourage top-down application design, using abstract function blocks to encapsulate system components.

Function blocks are like objects in object-oriented methodology, they use to encapsulate the behavior of an entity in the real world. For example, in electronic design they can represent an electronic device with inputs and outputs this is the same of the customer, employee, and product in a sells information system. They also provide an independent, interface oriented and component-based development. Each computational function block can be distributed over a wide range of computation components, such as PLC, Embedded Computer, or advanced Microcontroller.

To develop and run an IEC 61499 application there are some open source projects like Eclipse 4DIAC and FORTE. The 4DIAC is an open source IDE licensed by Eclipse Public License and provides PLC based programming environment under IEC 61499 Standard and partially IEC 61131-3 Standard. It uses FORTE to run and simulate applications developed by those standards. FORTE is also an open source project which has been developed by the same consortium which executes IEC 61499 Function Blocks.

\section{B. Virtual Reality Environment - Unity 3D}

There is many available software for 3D game development (Unity 3D C\#, Unreal Engine $\mathrm{C}++$, or Visionary Render the most used). Unity3D is chosen in our project due to its free pricing for development, portability, big supporting community, plenty of models and objects in the assets store, and the support for two of the most popular programming languages (JavaScript, C\#). Another reason for us choosing Unity $3 \mathrm{D}$ is because FORTE is $\mathrm{C}++$ based so the creation of the needed interfaces for the communication amongst the various components will be much easier. On top of the above a study conducted by Peters [16] also shows that Unity 3D is the most cost-effective, flexible and sustainable solution to develop VR/AR application. As someone can see from Fig. 1, the chosen Virtual/Augmented reality software will be the cornerstone component connecting all other components and port application for users.

\section{Use Cases}

The following two use case will be the basic ones to study how to build fault tolerant industrial application based on the proposed in [3] replication framework. The proposed 


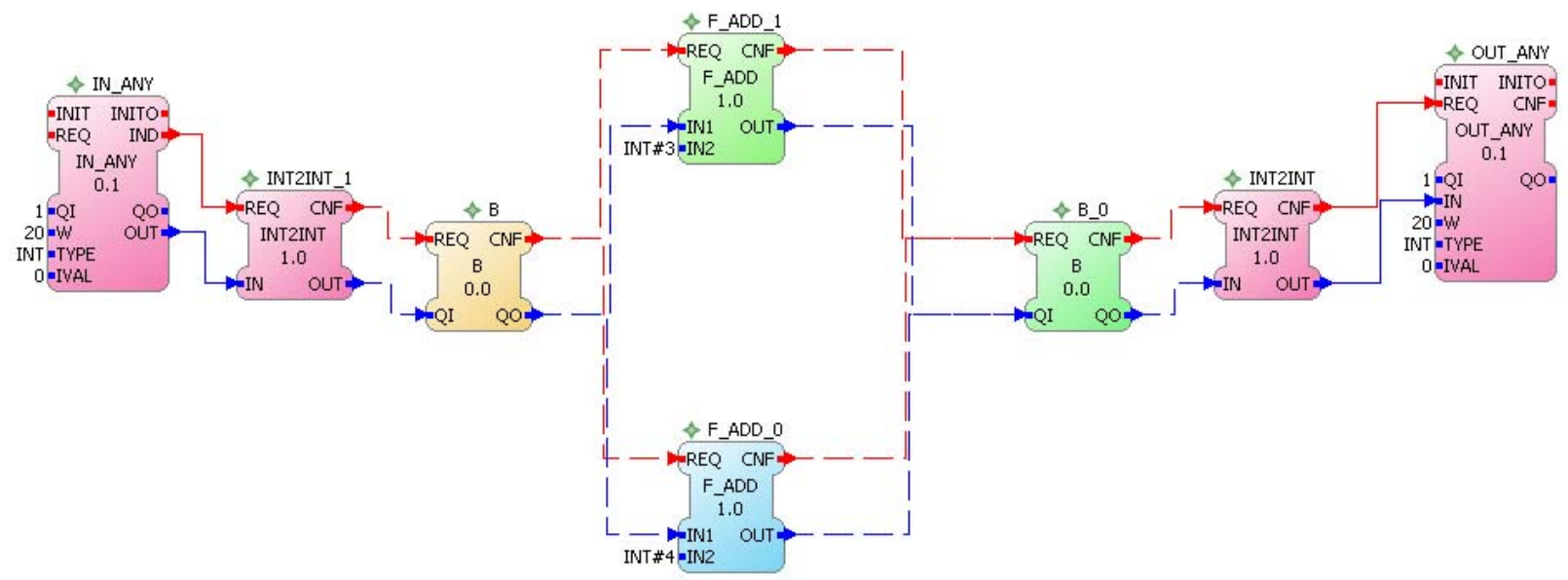

Fig. 2. Replication in XPlus3 example. [3]

replication framework was extensively studied and implemented in authors' previous work.

Some simple tests will be based on the trivial XPlus3 sample application that comes with the 4DIAC development environment. The original sample application (read input, add 3 , print result) simultaneously uses the FORTE and FBDK execution environments. FBDK is used in order to provide a graphical interface to the user (read input, print result), whereas FORTE is used to execute the FB containing the main application logic (add 3). This is possible since the FBs of the IEC 61499 application are distributed between two devices (or execution environments), and these FBs use communication SIFBs in each execution environment to exchange data and events.

Using the new replication layers a trivial replicated version of this application has been tested. In the replicated version (Fig. 3), the FB that adds a constant value to the input has been replicated. In principle, both replicas should add the same value, but in our test we are adding distinct values in each replica (3 and 4) so it becomes possible to identify which result was chosen by the voter.

Since the replication framework is being implemented solely for FORTE, all the replication related FBs must run on FORTE (and not FBDK). In other words, the FBDK device cannot execute the replication specific communication SIFBs, so the 'B' and 'B 0' were introduced to serve merely as intermediaries (or proxies) between the graphical user interface and the main replicated algorithm.

The sample replicated application therefore uses 5 devices (Fig. 3); one device runs on FBDK to provide the user interface (both input and output), whereas each of the other four devices is a distinct instantiation of the FORTE execution environment. One FORTE device runs the 'B' proxy $\mathrm{FB}$, another runs the other proxy FB 'B_0', and the remaining two FORTE instances each execute one of the replicas of the main FB ('F_ADD').

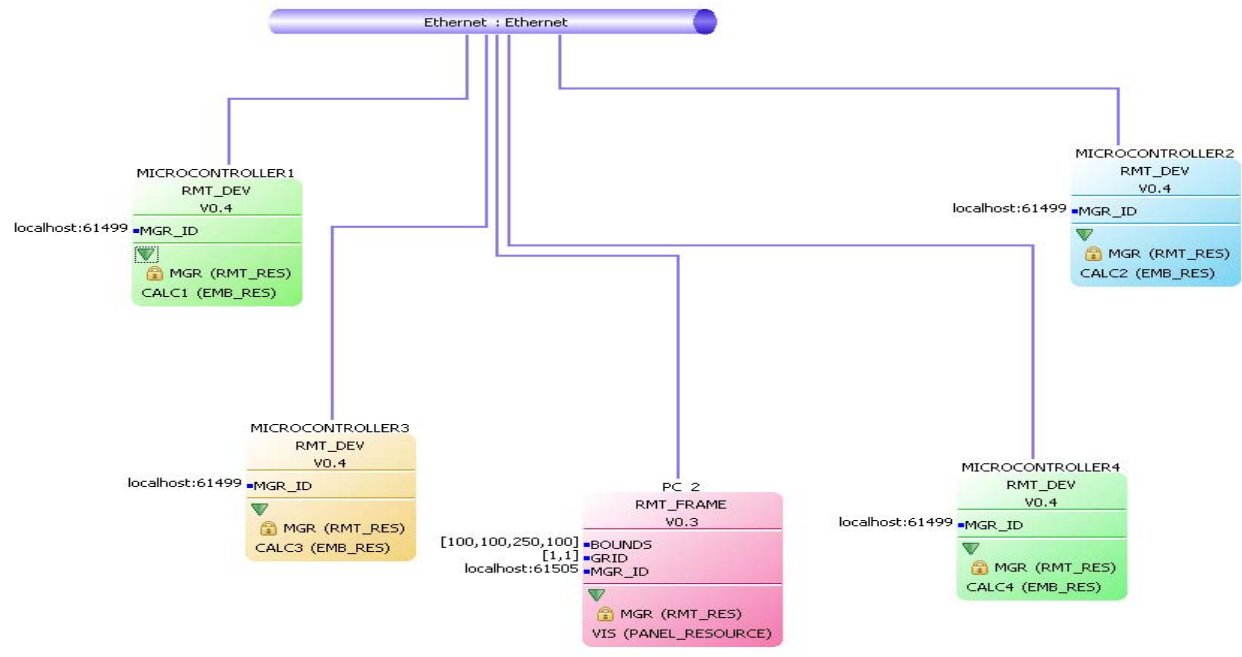

Fig. 3. System view and Communication Network in XPlus3 example. [3] 


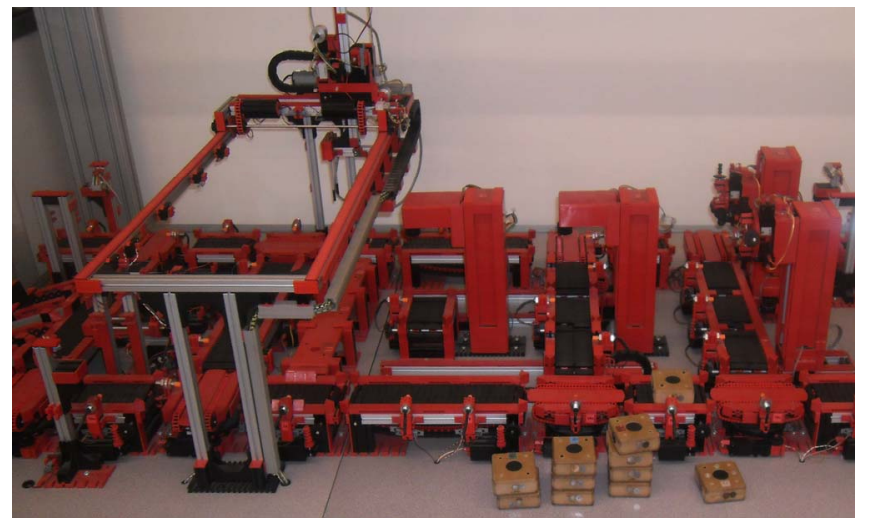

Fig. 4. Work-piece Transportation System. [3]

This trivial replicated application was successfully tested with all device instances running on the same computer. However, since communication between all instances is all done over a TCP/IP multicast network connection, we are convinced that the application would work the same were each FORTE and FBDK execution environment to run on a distinct hardware platform, with the platforms connected on a network.

Device failures were tested by simply stopping and starting each of the execution devices. The results were as expected, where the application was able to produce an output result as long as at least one of the replica devices were executing.

Another Use case will be an application to control the flow of work-pieces in a manufacturing cell (Fig. 4). This transport system is based on conveyors, and for simplicity of the control application we assume that work-pieces always follow a single direction: linear conveyors simply transfer pieces from one end to the other (action (i)), while rotating conveyors can rotate about their vertical axis, therefore either transferring the piece to one of two destinations (action (ii)) or receiving it from one of two origins (action (iii)) and transferring it to the same destination [3].

Every conveyor is assigned a FB, to be executed by a PLC type device. The most critical conveyors for the correct functioning of the whole transport chain are controlled by at least two PLC type devices, so that a possible failure in one device will not affect the functionality of the chain. In this paper we use just a small part of a transportation system to illustrate the replication functionality (Fig. 5).

This example contains 4 conveyors. Work-pieces enter this sub-system through $\mathrm{C} 0$, and are transferred to $\mathrm{C} 1$. From here, pieces can either go towards $\mathrm{C} 2$ or $\mathrm{C} 3$. The decision depends on the feedback the controller of $\mathrm{C} 1$ receives from the Distance Acceptance Stations residing on $\mathrm{C} 2$ and $\mathrm{C} 3$. The first one declaring that there is no work-piece in its visual field will be the one qualified to accept a new piece. Fig. 6 presents the FB networks controlling this physical layout.

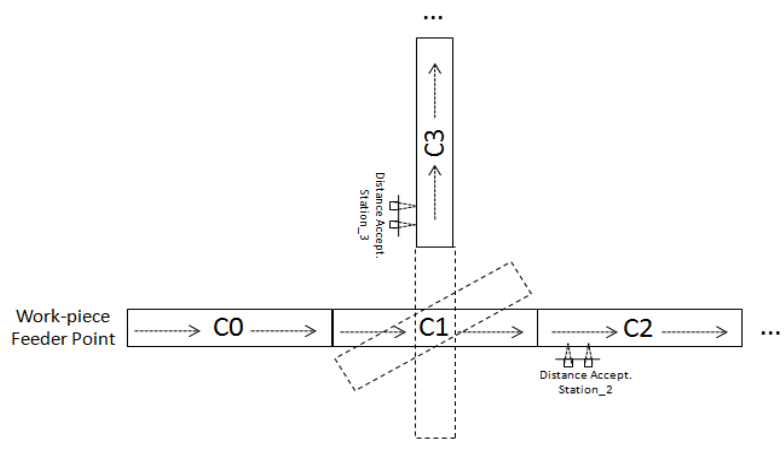

Fig. 5. Work-piece Transportation System. [3]

\section{VR Laboratory Setup}

In our premises, a new virtual engineering laboratory, carefully designed to help students become more industryaware and give them familiarity with new technologies to design, develop and test their ideas. The laboratory comprises five zones - the virtual reality auditorium, the project-based learning laboratory, the rapid prototyping and post-production suite, the digital factory and the head-mounted-display suite, which allows students to navigate their engineering projects in $3 \mathrm{D}$ using virtual reality headsets. Combined these zones allow students in LSBU's School of Engineering to perform detailed design reviews, rehearse in-depth training tasks, validate maintenance procedures or verify assembly and manufacturing processes at 1:1 scale. The technology used enables students to view their engineering projects in real-time, using the latest 3D modelling and visualization technologies. The available VR suite, its currently under two distinct systems.

- The first system has Nvisor ST50 and it is driven by Intersense IS 900 tracking (Ultrasound) and software engine Visionary Render and its script language is LUA. This system is mostly used for engineering testing/ training.

- The other system is the HTC Vives with its own tracking cameras (Infrared) and the available software are Unreal Engine, Unity 3D, and Autodesk Stingray LUA. This system is currently widely used in the industry and mostly recommended.

- There is also available a $6 \mathrm{~m} \times 4 \mathrm{~m}$ active wall, and back projection equipped with Mirage $4 \mathrm{k}$ projector.

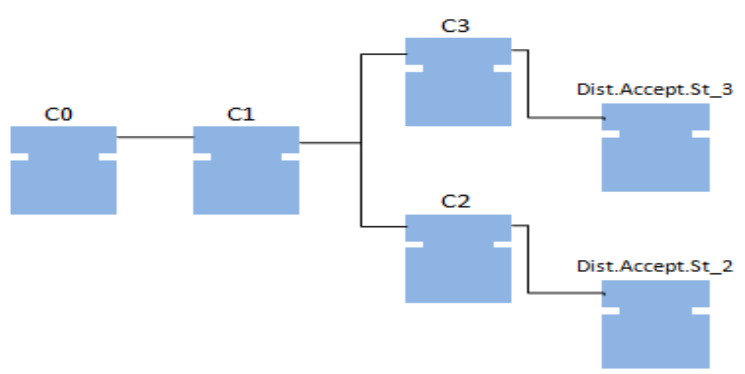

Fig. 6. FBs Connected Following the Physical Layout. [3] 


\section{CONCLUSION}

Viewing a project in virtual reality has many benefits to the engineer. Aside from gaining a greater understanding of how the project will work when fully realised, the engineer/educator/student can also spot any flaws or potential risks in the project, and correct these prior to further development. The main reasons that hinder wider use of Virtual Reality are the following: cost, time necessary for learning how to use hardware and software, possible health and safety effects, and reluctance to use and integrate the technology into a course or curriculum. As with all new technology, each of these issues will become less significant with time.

The authors in this work are presenting an initial framework for teaching industrial automations concepts, with the use of Virtual/Augmented reality to higher education students. The idea behind the proposed framework is to provide the ability to a Virtual/Augmented Reality user/student to easily build the automation system though the already provided IEC 61499 tools (FORTE and 4DIAC) and also provide him/her with the ability to parameterise the FBs' parameters to serve his/her needs. Another aspect is the capability to visualize the results with the use of examples available in a well build library. Examples like conveyors belt control and level control in a liquid's tanks. In order the above to be feasible, the needed interfaces for the communication amongst the Virtual/ Augmented Reality software and the IEC 61499 framework should be designed and developed and also the interfaces for the communication of the IEC 61499 to the actual use-cases should take place. For the steps to follow we will work towards a first prototype's implementation, to prove the feasibility of the proposed framework. This task is currently ongoing and hopefully we will present the results of our work in a next paper.

\section{ACKNOWLEDGMENT}

Professor Mario de Sousa from FEUP - Faculty of Engineering University of Porto helped in defining the initial use cases to be used, for building a replication framework based on IEC 61499 in [3], which will also be our initial use cases for the proposed Virtual/Augmented Reality framework.

\section{REFERENCES}

[1] International Electrotechnical Commission, "IEC61499-1 ed2.0 Function blocks - Part 1: Architecture", 2012-11-07

[2] FORDIAC \& FORTE: http://www.fordiac.org (accessed 2018/04/20).

[3] M. de Sousa, C. Chrysoulas, A. Homey, "Multiply and Conquer: A replication Framework for Building Fault Tolerant Industrial Applications.," INDIN 2015, 13th IEEE International Conference on Industrial Informatics, 22-24 July, 2015, Cambridge, UK.

[4] K. Abrosimova , "5 ways virtual reality will change education, Hypergrid Business," [Online], $2014 . \quad$ Available: https://www.hypergridbusiness.com/2014/09/5-ways-virtual-reality-willchange-education/

[5] H. Kaufmann, D. Schmalstieg \& M. Wagner, "Construct3D: A Virtual Reality Application for Mathematics and Geometry Education", Education and Information Technologies, Volume 5, Issue 4, pp. 263276. 2000.

[6] F. Mantovani, "VR learning: Potential and challenges for the use of 3D environments in education and training." In G. Riva \& C. Galimberti (Eds.), Towards cyberpsychology: Mind, cognitions and society in the internet age, pp. 207-226. Amsterdam: IOS Press, 2001.

[7] M. C. Salzman, C. Dede, R. B. Loftin and J. Chen, "A Model for Understanding How Virtual Reality Aids Complex Conceptual Learning," in Presence, vol. 8, no. 3, pp. 293-316, June 1999.

[8] Y. Chee, "Virtual reality in education: Rooting learning in experience." In Proceedings of the International Symposium on Virtual Education pp. 43-54. Busan, South Korea, 2001

[9] Z. Zacharia, "Using interactive simulations to enhance students' explanations regarding physical phenomena." [Online], 2003. Available: http://cblis.uniza.sk/cblis-cdold/2003/3.PartB/Papers/Computer_Based_Learning/Zacharia.pdf

[10] V. S. Pantelidis, "Reasons to Use Virtual Reality in Education and Training Courses and a Model to Determine When to Use Virtual Reality," Themes In Science And Technology Education, Special Issue, pp 59-70, 2009.

[11] R. Lewis and A. Zoitl, Modelling Control Systems Using IEC 61499. 2008.

[12] H. Chen, K. Feng, C. Mo, S. Cheng, Z. Guo, and Y. Huang, "Application of augmented reality in engineering graphics education." In IT in Medicine and Education (ITME), 2011 International Symposium on, vol. 2, pp. 362-365. IEEE, 2011

[13] J. I. Messner, S. C. Yerrapathruni, A. J. Baratta, and V. E. Whisker, "Using virtual reality to improve construction engineering education." In American Society for Engineering Education Annual Conference \&Exposition, 2003.

[14] S. Serafin, A. Adjorlu, N. Nilsson, L. Thomsen, and R. Nordahl, "Considerations on the use of virtual and augmented reality technologies in music education.” In 2017 IEEE Virtual Reality Workshop on K-12 Embodied Learning through Virtual Augmented Reality (KELVAR), pp. 1-4, March 2017. doi: 10.1109/KELVAR.2017.7961562

[15] D. Parmar, J. Isaac, S. V. Babu, N. D’Souza, A. E. Leonard, S. Jrg, K. Gundersen, and S. B. Daily. Programming moves: Design and evaluation of applying embodied interaction in virtual environments to enhance computational thinking in middle school students. In 2016 IEEE Virtual Reality (VR), pp. 131-140, March 2016. doi: 10.1109/VR .2016 .7504696

[16] E. Peters, B. Heijligers, J. de Kievith, X. Razafindrakoto, R. van Oosterhout, C. Santos, I. Mayer, and M. Louwerse, "Design for collaboration in mixed reality: Technical challenges and solutions." In $8^{\text {th }}$ International Conference on Games and Virtual Worlds for Serious Applications (VS-GAMES), pp. 1-7, Sept 2016. doi: 10.1109/VSGAMES.2016.7590343

[17] F. M. Dinis, A. S. Guimares, B. R. Carvalho, and J. P. P. Martins, "Virtual and augmented reality game-based applications to civil engineering education." In 2017 IEEE Global Engineering Education Conference (EDUCON), pp. 1683-1688, April 2017. doi: 10.1109/EDUCON.2017.7943075

[18] International Electrotechnical Commission, "IEC International Standard IEC 61131-3: Programmable Controllers, Part 3: Programming Languages", IEC, 2003. 